\title{
Valoración estadística - financiera para medio plazo del sector bancario en países con economías emergentes. El caso de Colombia
}

\author{
BOADA, ANTONIO JOSÉ \\ Escuela de Administración \\ CEIPA Business School. Medellín-Colombia \\ Correo electrónico: antonio.boada@ceipa.edu.co \\ MAYORCA, RÓMULO \\ Departamento de Formación General y Ciencias Básicas \\ Universidad Simón Bolívar. Camurí Grande. Estado Vargas (Venezuela) \\ Correo electrónico: rmayorca@usb.ve
}

\begin{abstract}
RESUMEN
Con el presente artículo se pretende exponer un proceso para la valoración de acciones de las empresas del sector bancario mediante múltiplos financieros empresariales, sustentado en variadas técnicas estadísticas tales como simulaciones de Montecarlo y modelos bayesianos de valoración continua de los indicadores relativos en el tiempo, con el fin de hacer proyecciones de escenarios lo más acertado posible en el mediano plazo. En cuanto a la metodología, el enfoque de la investigación fue cuantitativo, el tipo de estudio descriptivocorrelacional, y el diseño de la investigación fue no experimental. En virtud de que para llevar a cabo la valoración del desempeño y evolución de los múltiplos financieros en el tiempo, se deben considerar empresas similares, se decidió realizar previamente un análisis de cluster, para verificar posibles agrupaciones entre las entidades bancarias de acuerdo con las variables.
\end{abstract}

Palabras clave: múltiplos financieros, modelo lineal dinámico bayesiano, valoración de acciones, banca comercial, simulaciones.

Clasificación JEL: C15; C81; C51.

MSC2010: 62H30; 62F15. 


\title{
Statistical - financial value for the medium term of the banking sector in countries with emerging economies. The case of Colombia
}

\begin{abstract}
This article intends to expose a process for the valuation of shares of the companies of the banking sector through business financial multiples, based on various statistical techniques such as Monte Carlo simulations and Bayesian models of continuous valuation of the relative indicators over time, with in order to make scenario projections as successful as possible in the medium term. Regarding the methodology, the research approach was quantitative; the type of descriptive-correlational study, and the research design was non-experimental. Because in order to carry out the assessment of the performance and evolution of the financial multiples over time, similar companies must be considered, it was decided to previously conduct a cluster analysis, to verify possible groupings between the banking entities according to the variables.
\end{abstract}

Keywords: Financial Multiples, Bayesian Dynamic Linear Model, Stock Valuation, Commercial Banking, Simulations.

JEL classification: C15; C81; C51.

MSC2010: 62H30; 62F15.

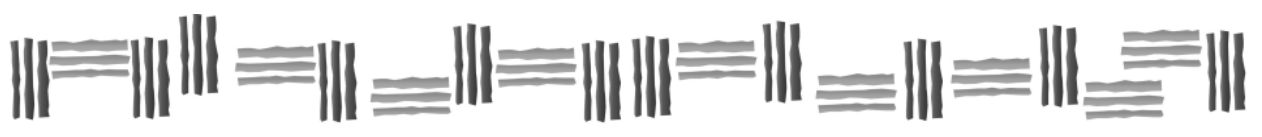




\section{Introducción.}

La valoración de una empresa para Fernández (2008) es "un ejercicio de sentido común que requiere unos pocos conocimientos técnicos y mejora con la experiencia” (p. 1). Esto no significa que sea una tarea simple, ya que valorar correctamente a una empresa y al precio de sus acciones en el mercado secundario, es una labor compleja. Por su parte, Boson, Cortijo y Flores (2009) comentan que la valoración de empresas está sujeta a cambios en los supuestos iniciales, según la magnitud de la decisión que se presente en casos como fusiones, venta (total o parcial), alianzas, reingeniería, liquidación o reorganización en momentos de crisis. Con relación a esto último, Londoño, Correa y Lopera (2014) indican que después de la crisis financiera de 2008 salieron a la luz los problemas internos de muchas instituciones financieras, en particular, para la evaluación del riesgo, siendo estos elementos que también influyen en la valoración de las empresas de cualquier sector.

Adicionalmente, Guijaro y Moya (2007) exponen que los modelos econométricos de valoración de empresas son diversos, pero todos han sido creados con la intención de tener una alta capacidad explicativa con el menor número de variables. Para García y García (2006), la evidencia empírica existente respecto a los modelos de valoración multifactoriales no es unánime en muchos ámbitos; sin embargo, es evidente el interés creciente que versa sobre la utilidad y el estudio de los modelos de valoración en los diferentes mercados y para los distintos activos financieros. En este mismo orden y dirección, Berk y De Marzo (2008) plantean que los modelos de valoración determinan las relaciones entre los flujos de efectivo futuros de la empresa, su costo de capital y el valor de sus acciones. Cabe destacar que para la investigación llevada a cabo, el universo objetivo de estudio fue el sector financiero, en particular, la banca comercial.

Al respecto, Ariza (2014) destaca que los bancos son entidades altamente apalancadas, lo que resulta en valoraciones mucho más dependientes de las cambiantes circunstancias económicas, en comparación con otros sectores. Dadas las consideraciones anteriores, aunque se han desarrollado diversos métodos de valoración, para el presente estudio se utilizó la técnica de múltiplos financieros derivados de los estados financieros - contables de las empresas del sector bancario que cotizan en las bolsas de valores. De acuerdo con Parra (2013), este método se fundamenta "en la observación que se hace de una empresa, a través de la aplicación de diversos indicadores, especialmente del mercado de valores de empresas con características similares para obtener un valor asociado a ella” (p. 89). En este mismo orden de ideas, Damodaran (2006), Demirakos, Strong y Walker (2004) señalan que los programas de análisis y los múltiplos de valoración son frecuentemente utilizados para estimar el valor de la empresa y justificar las recomendaciones de inversión.

Mediante la Ley del Precio Único, en un mercado competitivo, si dos o más activos son equivalentes, éstos tenderán a tener el mismo valor de mercado. Sin embargo, la gran pregunta radica en determinar este valor a los precios del mercado. Es por ello, que una de las principales técnicas de valoración corresponde a la de los múltiplos financieros, en donde a pesar de la diferencia entre los valores en libros (principios y métodos contables) y los valores de mercado (métodos financieros), los estados financieros auditados publicados y auditados a menudo ofrecen pistas en referencia a la situación financiera e información sobre su desempeño histórico que pueden ser importantes para el futuro.

Cabe agregar que Damodaran (2006) indica que dentro del mundo de las finanzas no es posible encontrar dos activos que sean iguales completamente. Por esta razón, para llevar a cabo una valoración se requiere utilizar activos similares a aquel cuyo valor se desea tasar, y que se establezca un criterio acerca de cuáles son aquellas diferencias que intervienen en el valor que tienen para los inversionistas. La valoración será adecuada siempre que exista una estabilidad en el desempeño de las operaciones empresariales, sin ningún tipo de anuncio relevante que origine 
tensión, reacción y saltos especulativos del mercado secundario, ante noticias de perspectivas futuras.

Hechas las consideraciones anteriores, es conveniente señalar que Land y Lang (2002), Zarowin (1990), Beaver y Morse (1978) indican que hay pruebas de que las diferencias contables son una fuente importante de variación en los múltiplos financieros.

Se debe resaltar que, por medio de la técnica de múltiplos financieros, fue posible ponderar los indicadores de cada empresa del sector bancario con base a corporaciones equivalentes del mismo sector. El procedimiento empleado consistió en simular escenarios que se ajustaran probabilísticamente de acuerdo al comportamiento histórico de los datos, para luego comparar ese resultado con el precio de la acción en el mercado secundario; esto permite establecer un valor que puede ser inhibidor o propulsor, dependiendo del comportamiento del mercado secundario en referencia al instrumento financiero considerado.

Este procedimiento potencia y complementa el método habitual de los múltiplos financieros con herramientas estadísticas, para la creación de un indicador porcentual el cual es utilizado para estimar el precio de la acción en el mercado secundario.

En este contexto, el objetivo general de este artículo es aplicar un conjunto de técnicas esquemáticamente desarrolladas para valorar el desempeño y evolución de los múltiplos financieros en el tiempo, para las empresas del sector bancario de Colombia.

\section{Metodología.}

El enfoque de la investigación fue cuantitativo, ya que se recolectaron datos para probar hipótesis con base en la medición numérica y el análisis estadístico para establecer patrones de comportamiento. Además, el tipo de estudio fue descriptivo-correlacional, porque lo que se buscó fue hacer una descripción y valoración de las acciones de las empresas del sector bancario con base a valores de empresas con características similares. El diseño fue no experimental ya que no se manipularon deliberadamente las variables.

A pesar de los avances teóricos y computacionales en la estadística bayesiana en las últimas décadas, poca atención se ha prestado a uno de los problemas más comunes en la investigación estadística (Gutiérrez y Zhang, 2009). La estadística bayesiana proporciona un enfoque teórico adecuado para el modelaje de datos, ya que permite manejar los problemas de actualización, de una forma más robusta que la estadística clásica, gracias al basamento axiomático y la flexibilización de cada problema en forma simultánea, permitiendo así desarrollar inferencias lo más coherente posible. En este sentido, para la fundamentación metodológica estadística de este caso, se alineó la técnica de acuerdo con Perichi (2002), West y Harrison (1989), en donde a través del Modelo Lineal Dinámico Bayesiano (MLDB), es posible determinar, a partir de datos históricos, el efecto producido por estas causas que originalmente no son contempladas por la Simulación de los Múltiplos Financieros; esto valorado a nivel de impacto potenciador o inhibidor del Indicador Relativo Logarítmico Comparativo (IRLC).

En este mismo orden de ideas, el MLDB se aplica sobre un Modelo de Suavización Exponencial del Indicador Relativo Logarítmico Comparativo IRLC [ $\ln (\operatorname{Pr}) / \ln (\mathrm{NVT})]$, calculado bajo una tasa $\alpha$, alineada al Porcentaje de Filtro Bayesiano $\left(\mathrm{A}_{\mathrm{t}}\right)$. La fórmula de Suavización Exponencial aplicado como estimador al Indicador Relativo Comparativo IRLC en tiempo "t" viene dada por la siguiente expresión:

$$
\widehat{I R L C}_{t}=\widehat{I R L C}_{t-1}+\left[\propto\left(I R L C_{t-1}-\widehat{I R L C}_{t-1}\right)\right]
$$


Por medio de este estudio, se procedió a generar una estructura estadística - financiera que permitiera establecer un procedimiento de valoración continua de instrumentos financieros, aplicable en instituciones como el Observatorio Financiero del CEIPA, Business School (Boada, 2016).

El procedimiento propuesto se desarrolla mediante fases, lo cual explica la sinergia realizada entre técnicas tradicionales financieras de valoración, en conjunto con modelos estadísticos y simulaciones probabilísticas que permiten crear estructuras de estimación consistente y valoración de escenarios futuros (Boada \& Gallego, 2016).

\subsection{Desarrollo de las Fases.}

Primera fase: se realizó el estudio inicial con la información suministrada por Bloomberg (Estados Financieros Consolidados y Auditados), desde el año 2010 hasta el 2016 de las empresas del sector bancario de Colombia, particularmente con los tres principales bancos del país: Davivienda, Banco de Bogotá y Bancolombia, a través de la información consolidada anualmente para cada organización.

Segunda fase: se generó una estructura de ponderación de los múltiplos financieros de cada empresa, fundamentado en los registros de las otras dos empresas competidoras. De esta manera es posible determinar, por ejemplo, el precio de la acción de Bancolombia en función de la relación del múltiplo financiero de Bancolombia y su asociación con los resultados de los competidores de tamaño similar, Davivienda y Banco de Bogotá.

Tercera fase: se calculó la proyección de las variables originales para los años futuros (patrimonio, beneficio neto, ingreso, EBITDA, precio de la acción en el mercado secundario, y añadiendo para el sector bancario las variables de EBITDA antes de provisión y pasivos), desde 2017 al 2021, calculando posteriormente los múltiplos financieros según estos resultados proyectados.

Cuarta fase: se corrieron cinco mil simulaciones de Montecarlo para cada uno de estos múltiplos financieros mediante la herramienta Risk Simulator, estableciendo un valor tentativo de cierre anual. Estas simulaciones se realizaron mediante distribuciones probabilísticas establecidas para cada variable de entrada, anteriormente descritas.

Quinta fase: se calcularon los indicadores relativos de comparación entre el valor teórico de los múltiplos financieros obtenidos a través de la simulación y el precio de la acción en el mercado secundario. De esta manera se estableció una proyección de tendencia mediante un modelo lineal dinámico bayesiano. Así mismo, también se puede valorar porcentualmente la percepción de la acción en el mercado secundario en correspondencia con las simulaciones efectuadas, determinando si el mercado se encuentra sobrevalorando o subvalorando a la empresa en función a la perspectiva matemática.

Sexta fase: se establecieron proyecciones para el precio de cada acción de las empresas consideradas del sector bancario colombiano, desde 2017 al 2021, valorados según las proyecciones de las variables de entrada: patrimonio, beneficio neto, ingreso, EBITDA, precio de la acción en el mercado secundario, y añadiendo para el sector bancario las variables de EBITDA antes de provisión y pasivos, simulación del valor teórico del múltiplo financiero mediante Risk Simulator y, finalmente, el indicador relativo de comparación establecido según el Modelo Lineal Dinámico Bayesiano.

Antes de presentar los resultados, es conveniente indicar que el sector bancario colombiano, se encuentra compuesto por seis bancos que cotizan en la Bolsa de Valores de Colombia (BVC): BBVA, AV villas, Occidente y los bancos tradicionales de mayor tamaño, que en conjunto abarcan el 70\% del mercado (Davivienda, Banco de Bogotá y Bancolombia). 
En virtud de que, para llevar a cabo la valoración del desempeño y evolución de los múltiplos financieros en el tiempo se deben considerar empresas similares, se decidió realizar previamente un análisis de cluster, para verificar posibles agrupaciones entre las entidades bancarias de acuerdo con las variables mencionadas con anterioridad.

El procedimiento seguido consistió en considerar una primera aproximación mediante un análisis de conglomerados jerárquicos, seleccionando el método de vinculación intergrupos y la distancia euclídea al cuadrado. La primera corrida permitió tener una solución preliminar con la conformación de cinco grupos. Lugo, se calcularon los centros iniciales para poder utilizar el método k-medias y obtener los grupos finales.

Es de resaltar que para la identificación de cada individuo se consideró la siguiente notación: BBVA (BB), AV villas (A), Occidente (O), Davivienda (D), Banco de Bogotá (B) y Bancolombia (BC). Como los datos recolectados fueron los correspondientes del 2010 al 2016, se estipuló que el banco por cada año era un individuo, así, D0 corresponde al banco Davivienda del año 2010, B1 corresponde al banco de Bogotá del año 2011, O6 corresponde al banco Occidente del año 2016 y así sucesivamente.

\section{Resultados.}

Con relación al análisis de cluster, en la figura 1 se presentan la distribución de los individuos y la conformación de los grupos finales.

Figura 1. Análisis de cluster para el Sector Bancario de Colombia que cotiza en la Bolsa de Valores BVC (Davivienda, Banco de Bogotá, Bancolombia Banco de Occidente, AV villas y BBVA).

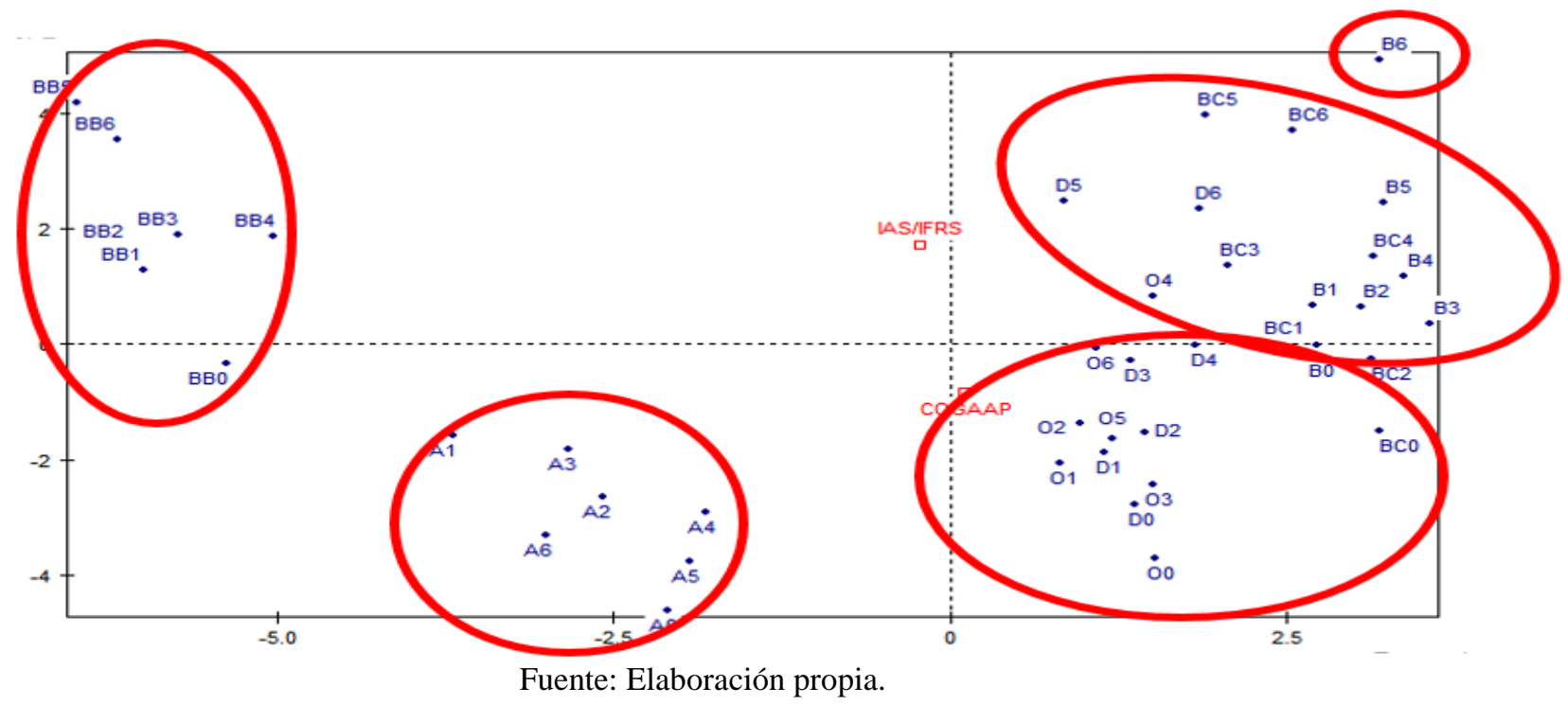

En la figura 1 se denota estadísticamente la creación de cinco tipos de cluster o conglomerados, perfectamente establecidos, y que pueden tener un nivel interesante de interpretación y análisis que detallamos:

- Grupo 1. Agrupa Davivienda en su época joven (2010, 2011, 2012, 2013 y 2014), Bancolombia en 2010 y Banco de Occidente en casi su totalidad (2010, 2011, 2012, 2013, 2015 y 2016). Este grupo refleja lo que se denominó "Bancos en Período de Crecimiento". Para este cluster, Davivienda se consideraba un banco de poca participación de mercado, pero ya para 2015 y 2016 se consolidó como una entidad financiera de importante tamaño y el tercero del sector bancario colombiano. Así mismo, 
se observa que Banco de Occidente poco a poco está aumentando sus indicadores, y pronto pasará a consolidarse como el cuarto banco de importancia relevante para el país.

- Grupo 2. Corresponde al grupo más grande, agrupa Davivienda en su época actual (2015, y 2016), Banco de Bogotá en casi todos los años (2010, 2011, 2012, 2013, 2014 y 2015), la totalidad de Bancolombia (2011, 2012, 2013, 2014, 2015, 2016) y como un caso excepcional, banco de occidente sólo en 2014. Este grupo refleja lo que se denominó como "Bancos Consolidados en el Mercado". Para este conglomerado, se observan los principales bancos del sector en Colombia, así como su estabilidad y solidez en el tiempo. También se valora a Banco de Occidente como el potencial que posee para entrar en este grupo en el mediano plazo.

- Grupo 3. Corresponde al grupo más pequeño, agrupa solamente al Banco de Bogotá en el último año 2016. Este grupo, refleja lo que se denominó como "Súper Bancos". Para este conglomerado, se estima que sea la evolución de los bancos, una vez que se incluyan los beneficios obtenidos en las sucursales que posee en el exterior. Ahora con las normas NIFF, Banco de Bogotá puede reflejar en sus estados financieros los beneficios obtenidos por sus sucursales en el exterior (para aquellas entidades que posean dicha inversión). Banco de Bogotá aparenta ser el primer banco de Colombia que llega a este nivel de estructura bancaria.

- Grupo 4. Agrupa solamente a la entidad bancaria de AV villas en todos los años (2010, 2011, 2012, 2013, 2014, 2015 y 2016). Este grupo, reflejan lo que se denominó como "Bancos Pequeños". Para este conglomerado, se analiza que Av villas representa el banco más pequeño de los seis analizados, así mismo su nivel de Beneficio y EBITDA en función a los ingresos se expone bastante reducido al compararlo con las restantes entidades financieras.

- Grupo 5. Finalmente, el quinto grupo expone solamente a la entidad bancaria de BBVA en todos los años (2010, 2011, 2012, 2013, 2014, 2015 y 2016). Este grupo refleja lo que se denominó como "Bancos Pequeños con Alto Endeudamiento". Para este conglomerado, se analiza que BBVA representa un banco bajo el mismo nivel de patrimonio que el Banco de Occidente, pero su nivel de endeudamiento (pasivo) se encuentra a un nivel elevado cuando lo comparamos con sus activos totales.

Primera Fase. Estudio inicial con la información suministrada por Bloomberg (Estados Financieros Consolidados y Auditados), desde el año 2010 hasta el 2016 de las empresas del sector bancario de Colombia: Davivienda, Banco de Bogotá y Bancolombia. Se procedió a calcular los múltiplos financieros para la información histórica registrada en los años 2010, 2011, 2012, 2013, 2014, 2015 y 2016, elaborando los gráficos respectivos (figura 2) con la información recabada, se puede observar el comportamiento de cada una de las variables para las tres empresas consideradas del sector bancario colombiano. 
Figura 2. Información de las tres empresas del sector bancario colombiano.

\section{PATRIMONIO}

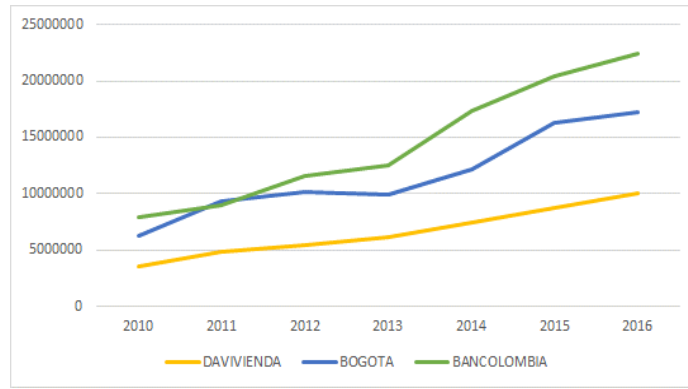

\section{INGRESO}

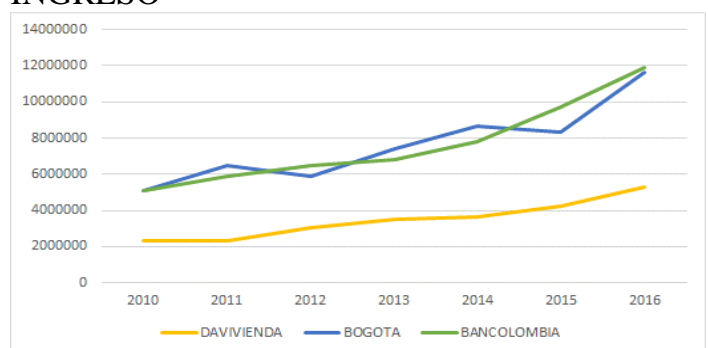

\section{EBITDA}

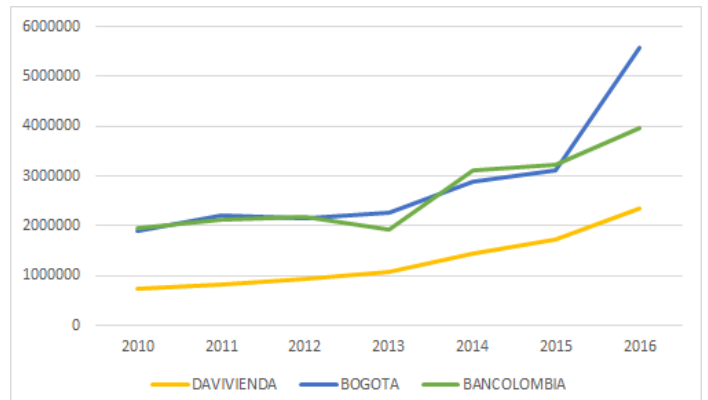

\section{BENEFICIO NETO}

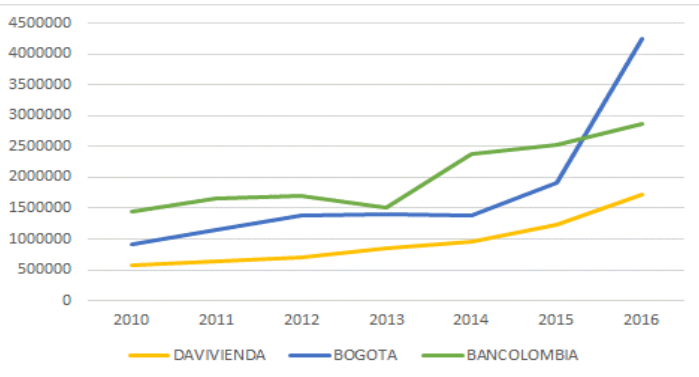

\section{PRECIO ACCIÓN}

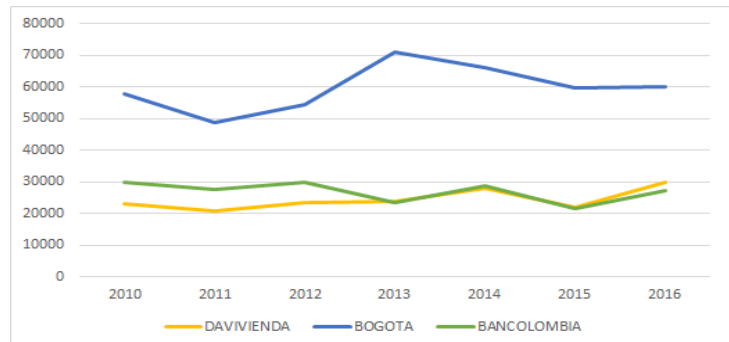

EBITDA ANTES DE PROVISIÓN

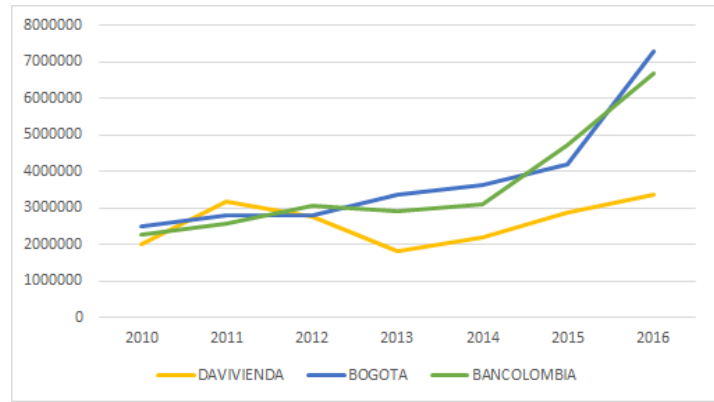

\section{PASIVOS TOTALES}

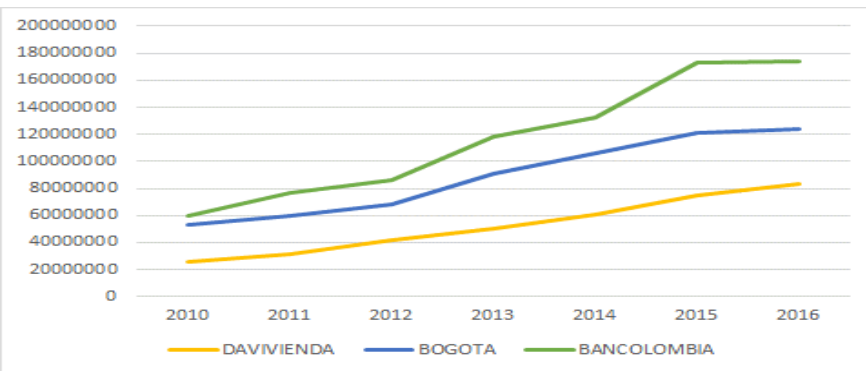

Fuente: Elaboración propia - Bloomberg.

Segunda Fase. Generación de una estructura de ponderación de los múltiplos financieros de cada banco, fundamentado en los registros de los competidores. Estos múltiplos financieros fueron calculados en su forma directa y, posteriormente, también se procedió al cálculo de su forma como múltiplo secundario según el precio de la acción en el mercado secundario. Con el valor expuesto de estos múltiplos financieros para cada banco durante cada año, fue posible calcular la "Valoración Teórica del Banco", o múltiplo general calculado a partir de los múltiplos financieros de las compañías competidoras del sector (tabla 1). 
Tabla 1. Valores Teóricos según los Múltiplos Financieros (antes de simulaciones de Montecarlo), para los bancos Davivienda, Banco de Bogotá y Bancolombia (calculado con base a sus indicadores financieros comparables).

\begin{tabular}{lcccc}
\hline \multicolumn{1}{c}{ EMPRESA } & AÑO & $\begin{array}{c}\text { Ln (VALOR } \\
\text { PROMEDIO } \\
\text { TEÓRICO) }\end{array}$ & $\begin{array}{c}\text { VALOR PROMEDIO } \\
\text { TEÓRICO según } \\
\text { Múltiplos Financieros }\end{array}$ & $\begin{array}{c}\text { Precio Real de la } \\
\text { acción para fin de año }\end{array}$ \\
\hline Davivienda & 2010 & 10,07588178 & $23.762,92$ & $22.980,00$ \\
Davivienda & 2011 & 10,05033259 & $23.163,48$ & $20.860,00$ \\
Davivienda & 2012 & 10,11236112 & $24.645,78$ & $23.480,00$ \\
Davivienda & 2013 & 10,06168026 & $23.427,83$ & $23.660,00$ \\
Davivienda & 2014 & 10,10420815 & $24.445,66$ & $28.100,00$ \\
Davivienda & 2015 & 9,927980511 & $20.495,90$ & $21.800,00$ \\
Davivienda & 2016 & 10,00874651 & $22.219,96$ & $30.000,00$ \\
Banco de Bogotá & 2010 & 11,30766549 & $81.443,55$ & $57.639,91$ \\
Banco de Bogotá & 2011 & 11,27646627 & $78.941,80$ & $48.779,89$ \\
Banco de Bogotá & 2012 & 11,27905183 & $79.146,18$ & $54.255,19$ \\
Banco de Bogotá & 2013 & 11,22790373 & $75.199,79$ & $71.178,82$ \\
Banco de Bogotá & 2014 & 11,35175766 & $85.114,92$ & $66.100,00$ \\
Banco de Bogotá & 2015 & 11,02083716 & $61.134,83$ & $59.500,00$ \\
Banco de Bogotá & 2016 & 11,52252572 & $100.964,64$ & $60.200,00$ \\
Bancolombia & 2010 & 10,04288186 & $22.991,54$ & $29.940,00$ \\
Bancolombia & 2011 & 9,884482743 & $19.623,49$ & $27.620,00$ \\
Bancolombia & 2012 & 9,990490377 & $21.817,99$ & $29.820,00$ \\
Bancolombia & 2013 & 10,04470998 & $23.033,61$ & $23.440,00$ \\
Bancolombia & 2014 & 10,22186025 & $27.497,77$ & $28.640,00$ \\
Bancolombia & 2015 & 10,03572654 & $22.827,62$ & $21.600,00$ \\
Bancolombia & 2016 & 10,11128657 & $24.619,31$ & $27.180,00$ \\
\hline
\end{tabular}

Fuente: Elaboración propia.

Es importante destacar que esta fase dos genera la estructura fundamental en la que se basará la simulación de Montecarlo, una vez se definan las distribuciones probabilísticas para las variables aleatorias utilizadas: patrimonio, beneficio neto, ingreso, precio de la acción en el mercado secundario, EBITDA, EBITDA antes de provisión y pasivos.

Tercera Fase. Proyección de las variables y datos para los años futuros, desde 2017 al 2021, calculando los múltiplos financieros según los resultados proyectados.

Para esta fase, tras el análisis del comportamiento histórico de las variables utilizadas de patrimonio, ingreso, beneficio, precio de la acción, EBITDA, EBITDA antes de provisión y pasivo, se procedió a realizar una proyección de valores futuros, fundamentados en las técnicas de regresión y de Movimiento Browniano Exponencial o Geométrico.

Para esta técnica, la volatilidad dependerá de la fluctuación promedio histórica de los resultados obtenidos en los balances generales analizados previamente. De esta manera, después de aplicar las técnicas de modelo de regresión y pronósticos de procesos estocásticos, las estimaciones de estas variables para los años 2017 a 2021 se presentan en la tabla 2.

Tabla 2. Predicciones de variables de entrada para los bancos Davivienda, Banco de Bogotá y Bancolombia. 


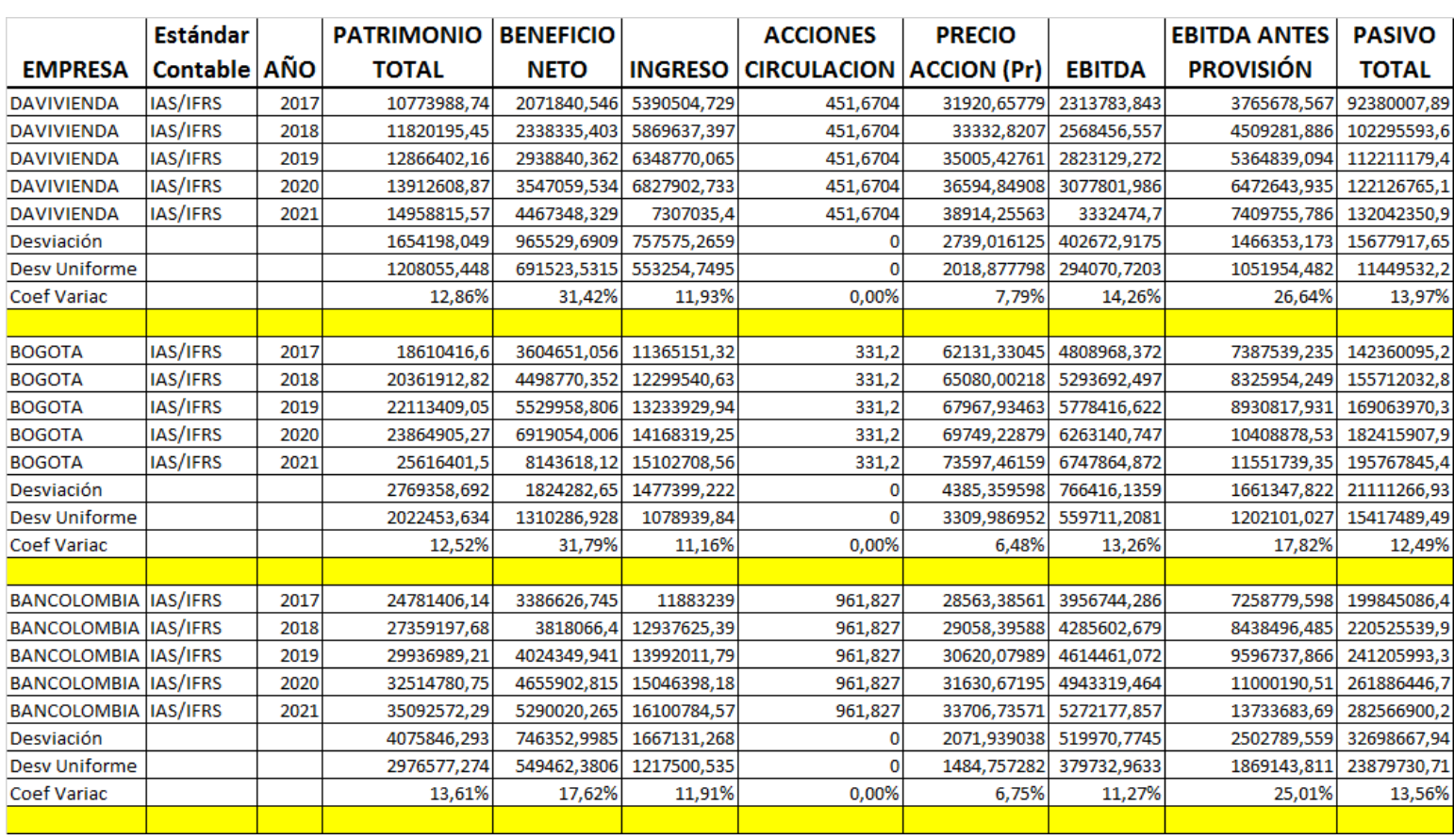

Fuente: Elaboración propia.

Las proyecciones directas, expresan únicamente una opción basada en probabilidades de acuerdo al comportamiento histórico de los balances generales y estados de ganancias y pérdidas para cada empresa del sector bancario colombiano, auditados y publicados por Bloomberg; no obstante, aún se deben realizar estimaciones del valor teórico del múltiplo financiero en base a simulaciones de distribuciones probabilísticas ( $4^{\mathrm{a}}$ fase) y luego estimar y proyectar un indicador relativo de comparación entre este valor teórico y el precio de la acción en el mercado secundario ( $5^{\mathrm{a}}$ y $6^{\mathrm{a}}$ fase).

Cuarta Fase. Ejecución de simulaciones para cada uno de los valores teóricos derivados de los múltiplos financieros mediante la herramienta Risk Simulator, estableciendo un valor tentativo de cierre anual.

Para esta fase, se corrieron cinco mil simulaciones de Montecarlo en cada uno de los valores teóricos anuales, derivados de los múltiplos financieros para los tres principales bancos de Colombia (Davivienda, Banco de Bogotá y Bancolombia). Este proceso, se realizó definiendo distribuciones probabilísticas y parámetros para cada una de las siete variables a simular (patrimonio, beneficio neto, ingreso, precio de la acción en el mercado secundario, EBITDA, EBITDA antes de provisión y pasivo), ejecutando finalmente la simulación mediante la herramienta Risk Simulator, obteniendo así un valor tentativo de cierre anual.

De esta manera, tras establecer los modelos de regresión para el patrimonio, ingresos, EBITDA y pasivos, así como también los procesos estocásticos de caminata aleatoria exponencial para estimar los valores de beneficio, EBITDA antes de provisión y precio de la acción, fue posible construir una tabla con los valores de predicción para el valor teórico del múltiplo financiero de las tres empresas del sector bancario de Colombia en función a dichas variables de interés.

Entre las múltiples distribuciones probabilísticas reflejadas por la aplicación de Risk Simulator, esta investigación se centró en tres distribuciones probabilísticas:

1. Distribución Probabilística Uniforme: de acuerdo con Lind (2012) para la distribución uniforme, todos los valores contenidos entre los mínimos y máximos suceden con la misma probabilidad. Esta distribución es utilizada para campos altamente fluctuantes y sin posibilidad 
de conocimiento ni estimación previa de su comportamiento o tendencia. Para el presente estudio, se utilizó esta distribución de probabilidad para el beneficio neto y para el precio de cotización por acción, ya que ambas variables en su condición de alto nivel de fluctuación y ausencia de patrones establecidos, pueden generar cualquier tipo de relación en mercados con economías emergentes. Para utilizar esta distribución probabilística, fue necesario establecer los valores de máximos y mínimos, según el comportamiento del resultado de cada banco a nivel histórico o proyectado, y una desviación promedio estable para una distribución uniforme de cada una de las tres empresas en cada una de las dos variables (beneficio y precio de la acción).

La fórmula de cálculo para la desviación típica de la distribución es:

$$
\hat{\sigma}=\sqrt{\frac{\left[\operatorname{Max}\left(x_{i}\right)-\operatorname{Min}\left(x_{i}\right)\right]^{2}}{12}}
$$

El valor mínimo y el valor máximo son representados según los valores registrados históricamente y/o proyectados según cada una de las dos variables y cada una de las tres empresas, ampliados según tres veces la desviación estándar previamente calculados (Resultado Empresa \pm 3 . Desviación Estándar Empresa), con la finalidad de contemplar el 99,70\% de los casos posibles.

2. Distribución Probabilística Triangular: es un modelo de distribución de probabilidad continua, que tiene por función de densidad la estructura siguiente:

$$
f X(x \mid a, m, b)=\frac{2}{b-a} * \begin{cases}\frac{x-a}{m-a} & a \leq x \leq m \\ \frac{b-x}{b-m} & m \leq x \leq b\end{cases}
$$

Esta distribución presenta tres parámetros y de acuerdo con Herrerías y Herrerías (2009), describe una situación donde se conocen los valores mínimo (a), máximo (b) y el que con mayor probabilidad puede suceder (c); variando la posición más probable con relación a los extremos, la distribución puede ser simétrica o no. Es de uso frecuente en la toma de decisiones empresariales, para modelar procesos estocásticos o de riesgo comercial y fue utilizada en el presente estudio para simular la variable de EBITDA antes de provisión, ya que la estimación realizada por el movimiento browniano exponencial, exponía una estimación con mayor probabilidad que los extremos.

3. Distribución Probabilística Logarítmica Normal: también es conocida como ley del efecto proporcional. Su función de densidad viene dada por la siguiente estructura:

$$
g(y)=\frac{1}{\sqrt{2 \pi} \sigma} \frac{1}{y} \exp \left(-\frac{(\log y-\mu)^{2}}{2 \sigma^{2}}\right) \quad y \geq 0
$$

De acuerdo con Sandoval (2005), la distribución lognormal es frecuentemente utilizada para expresar el comportamiento de observaciones con asimetría positiva, por ejemplo, en un análisis financiero, para evaluar una propiedad en bienes raíces o el análisis de precio de acciones y en todos aquellos fenómenos donde los valores observados no pueden caer por debajo de cero. Los precios resultantes muestran esta tendencia porque no pueden caer por debajo del límite menor de cero, pero pueden incrementar su precio indefinidamente. Esta distribución fue utilizada para alimentar las simulaciones del patrimonio, ingreso, EBITDA y pasivo; términos financieros que presentan variabilidad controlada, pero no pueden ser negativos.

\subsection{Simulador de Montecarlo.}

El Montecarlo es un método numérico que permite resolver problemas físicos y matemáticos mediante la simulación de variables aleatorias. De acuerdo con García (2003) la clave de la 
simulación de Montecarlo consiste en crear un modelo matemático del sistema, proceso o actividad que se quiere analizar, identificando aquellas variables cuyo comportamiento aleatorio determina el comportamiento global del sistema. Mediante el simulador de Montecarlo de Risk Simulator, se realizaron cinco mil simulaciones de escenarios, en donde se establecieron criterios probabilísticos de fluctuación para cada variable original. De esta manera, se identificó la probabilidad que separa el valor teórico proporcionado por los múltiplos financieros y el valor real obtenido por el mercado secundario de la Bolsa de Valores. En la tabla 3 se presentan los resultados de los valores teóricos obtenidos.

Tabla 3. Valor Teórico de la empresa obtenido por Múltiplos Financieros. A nivel directo (sin simulación) y con simulación de Montecarlo, para los bancos Davivienda, Banco de Bogotá y Bancolombia.

\begin{tabular}{lcccc}
\hline & AÑ & $\begin{array}{c}\text { VALOR } \\
\text { TEÓRICO según } \\
\text { múltiplos } \\
\text { financieros con } \\
\text { información } \\
\text { histórica }\end{array}$ & $\begin{array}{c}\text { NUEVO VALOR TEÓRICO } \\
\text { (NTV), según múltiplos } \\
\text { financieros y simulación de } \\
\text { Montecarlo (con información }\end{array}$ & $\begin{array}{c}\text { Precio real (Pr) de la } \\
\text { acción en el mercado } \\
\text { secundario de la } \\
\text { bolsa de valores }\end{array}$ \\
EMPRESA & Aistórica) & \\
\hline Davivienda & 2010 & 23762,92 & $30.263,57$ & 22980,00 \\
Davivienda & 2011 & 23163,48 & $28.257,10$ & 20860,00 \\
Davivienda & 2012 & 24645,78 & $29.522,26$ & 23480,00 \\
Davivienda & 2013 & 23427,83 & $25.044,26$ & 23660,00 \\
Davivienda & 2014 & 24445,66 & $27.135,55$ & 28100,00 \\
Davivienda & 2015 & 20495,90 & $21.899,08$ & 21800,00 \\
Davivienda & 2016 & 22219,96 & $22.227,82$ & 30000,00 \\
Banco de Bogotá & 2010 & 81443,55 & $110.227,31$ & 57639,91 \\
Banco de Bogotá & 2011 & 78941,80 & $101.316,04$ & 48779,89 \\
Banco de Bogotá & 2012 & 79146,18 & $102.129,82$ & 54255,19 \\
Banco de Bogotá & 2013 & 75199,79 & $95.798,28$ & 71178,82 \\
Banco de Bogotá & 2014 & 85114,92 & $108.934,28$ & 66100,00 \\
Banco de Bogotá & 2015 & 61134,83 & $79.268,81$ & 59500,00 \\
Banco de Bogotá & 2016 & 100964,64 & $114.530,91$ & 60200,00 \\
Bancolombia & 2010 & 22991,54 & $29.827,92$ & 29940,00 \\
Bancolombia & 2011 & 19623,49 & $24.413,71$ & 27620,00 \\
Bancolombia & 2012 & 21817,99 & $27.883,77$ & 29820,00 \\
Bancolombia & 2013 & 23033,61 & $33.560,33$ & 23440,00 \\
Bancolombia & 2014 & 27497,77 & $38.800,95$ & 28640,00 \\
Bancolombia & 2015 & 22827,62 & $30.742,46$ & 21600,00 \\
Bancolombia & 2016 & 24619,31 & $34.348,03$ & 2780,00 \\
\hline
\end{tabular}

Fuente: Elaboración propia.

El valor teórico obtenido para cada empresa según los múltiplos comparables, expone el Valor Teórico de cada banco en comparación con las otras dos empresas comparables.

Quinta Fase. Cálculo de los indicadores relativos de comparación entre el Nuevo Valor Teórico de los múltiplos financieros obtenidos a través de la simulación y el Precio de la Acción (Pr) en el mercado secundario. De esta forma, se establece una proyección de tendencia mediante un modelo lineal dinámico bayesiano.

Una vez que se obtienen el nuevo valor teórico de una empresa con base a los múltiplos financieros de sus empresas competidoras en el mismo sector (bancario de Colombia), se procede 
a establecer un indicador relativo comparativo con el precio de la acción en el mercado secundario. Por esta vía, es posible valorar el comportamiento de este indicador, generando un Modelo Lineal Dinámico Bayesiano, que permita estimar el valor de este indicador de ajuste comparativo en el tiempo para cada una de las empresas del sector bancario colombiano. Los resultados se visualizan en la figura 3.

Figura 3. Indicador Relativo Logarítmico Comparativo IRLC $[\ln (\operatorname{Pr}) / \ln (\mathrm{NVT})]$ para los bancos Davivienda, Banco de Bogotá y Bancolombia.

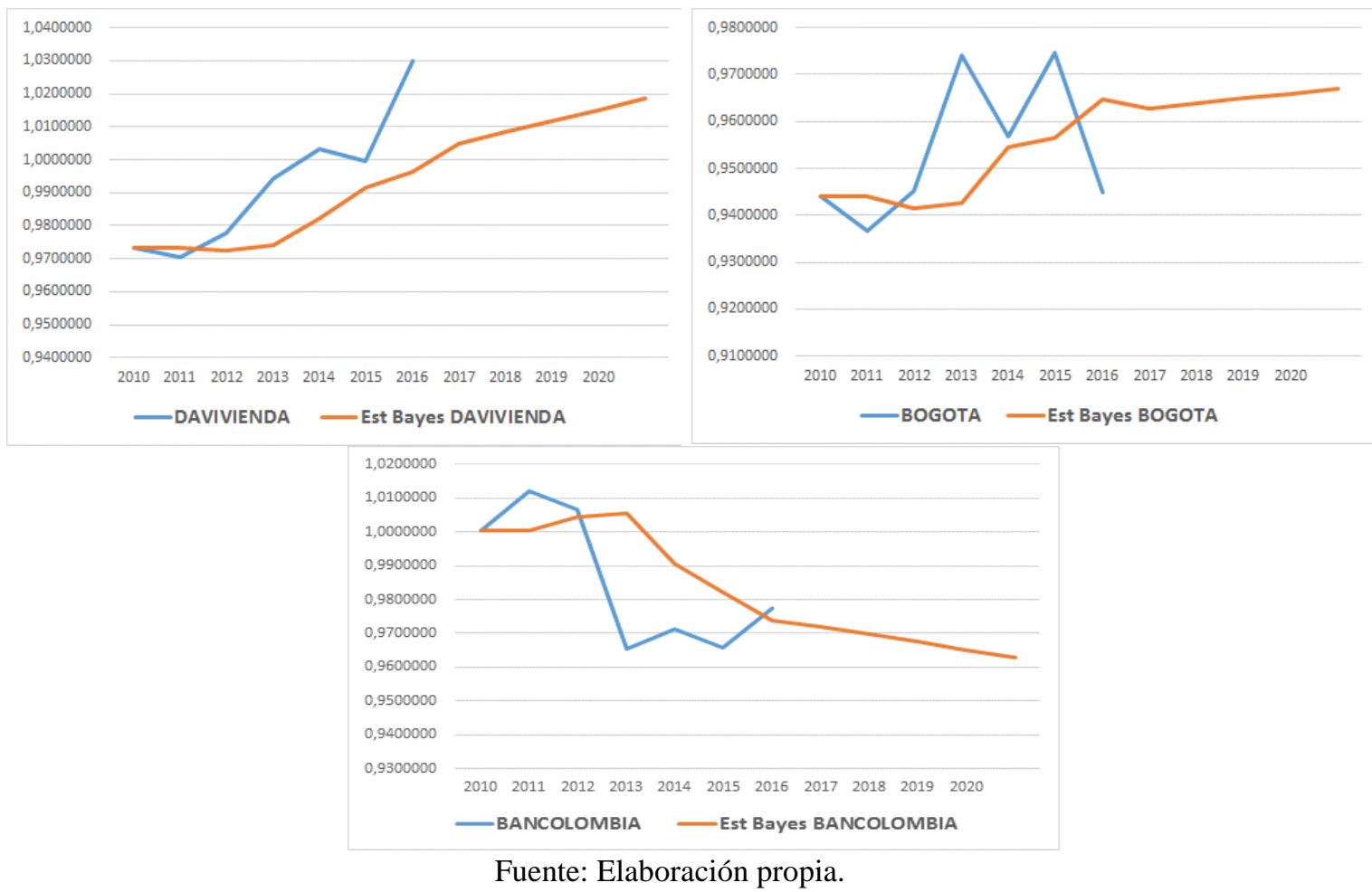

Sexta Fase. Proyecciones para el precio de cada acción de las empresas del sector bancario colombiano en el mercado secundario, desde 2017 al 2021, valorados según las proyecciones de las variables de entrada: beneficio, patrimonio, ingresos, precio de la acción, EBITDA, EBITDA antes de provisión y pasivo; simulación del valor teórico del múltiplo financiero mediante Risk Simulator (Nuevo Valor Teórico NVT) y, finalmente, el Indicador Relativo Logarítmico de Comparación IRLC establecido según el modelo lineal dinámico bayesiano.

Por limitaciones en la consolidación de Bloomberg, la información trabajada fue de periodicidad anual, desde el año 2010 en adelante. En este sentido, para este artículo se procedió a correr simulaciones, fundamentada en la información obtenida 2010 - 2016. Los resultados se presentan en la tabla 4. 
Tabla 4. Precio Proyectado de la Acción, para las empresas del sector bancario de Colombia (Davivienda, Banco de Bogotá y Bancolombia).

\begin{tabular}{|c|c|c|c|c|c|}
\hline EMPRESA & AÑo & $\begin{array}{l}\text { NUEVO VALOR TEÓRICO (NVT) } \\
\text { según Múltiplos Financieros y } \\
\text { Simulación de Montecarlo (CON } \\
\text { INFORMACIÓN PROYECTADA) }\end{array}$ & $\begin{array}{c}\text { Indicador Relativo } \\
\text { Logarítmico } \\
\text { Comparativo IRLC } \\
{[\ln (\mathbf{P r}) / \ln (\mathrm{NVT})]}\end{array}$ & $\begin{array}{l}\text { Modelo Lineal } \\
\text { Dinámico } \\
\text { Bayesiano para } \\
\text { Proyectar el IRLC }\end{array}$ & $\begin{array}{l}\text { PRECIO DE LA } \\
\text { ACCIÓN } \\
\text { PROYECTADO }\end{array}$ \\
\hline DAVIVIENDA & 2010 & & 0,973315715 & 0,973315715 & - \\
\hline DAVIVIENDA & 2011 & & 0,970386544 & 0,973315715 & - \\
\hline DAVIVIENDA & 2012 & & 0,977752069 & 0,972287416 & - \\
\hline DAVIVIENDA & 2013 & & 0,994386195 & 0,974234035 & - \\
\hline DAVIVIENDA & 2014 & & 1,003421121 & 0,982105066 & - \\
\hline DAVIVIENDA & 2015 & & 0,999546262 & 0,991325959 & - \\
\hline DAVIVIENDA & 2016 & & 1,029958004 & 0,996269187 & - \\
\hline DAVIVIENDA & 2017 & $24.277,37$ & & 1,004976491 & $25.528,46$ \\
\hline DAVIVIENDA & 2018 & $24.666,47$ & & 1,008332979 & $26.835,29$ \\
\hline DAVIVIENDA. & 2019 & $26.662,14$ & & 1,01169266 & $30.036,24$ \\
\hline DAVIVIENDA & 2020 & $27.631,18$ & & 1,015054046 & $32.230,01$ \\
\hline DAVIVIENDAA & 2021 & $29.789,16$ & & 1,018416341 & $36.012,57$ \\
\hline BOGOTA & 2010 & & 0,944159108 & 0,944159108 & - \\
\hline BOGOTA & 2011 & & 0,936584541 & 0,944159108 & - \\
\hline BOGOTA & 2012 & & 0,945158136 & 0,941500022 & - \\
\hline BOGOTA & 2013 & & 0,974102056 & 0,942622938 & - \\
\hline BOGOTA & 2014 & & 0,956927536 & 0,954645793 & - \\
\hline BOGOTA & 2015 & & 0,974569756 & 0,956605786 & - \\
\hline BOGOTA & 2016 & & 0,944785436 & 0,964877532 & - \\
\hline BOGOTA & 2017 & $113.210,02$ & & 0,96275784 & $73.395,14$ \\
\hline BOGOTA & 2018 & $116.727,80$ & & 0,963834876 & $76.545,44$ \\
\hline BOGOTA & 2019 & $122.884,48$ & & 0,964912938 & $81.455,71$ \\
\hline BOGOTA & 2020 & $127.886,70$ & & 0,965991546 & $85.733,42$ \\
\hline BOGOTA & 2021 & $135.591,23$ & & 0,967070445 & $91.881,80$ \\
\hline BANCOLOMBLA & 2010 & & 1,000364028 & 1,000364028 & - \\
\hline BANCOLOMBLA & 2011 & & 1,012213862 & 1,000364028 & - \\
\hline BANCOLOMBIA & 2012 & & 1,006558802 & 1,004523966 & - \\
\hline BANCOLOMBLA & 2013 & & 0,965560185 & 1,005664151 & - \\
\hline BANCOLOMBIA & 2014 & & $0,97126304 \mathrm{~S}$ & 0,990657983 & - \\
\hline BANCOLOMBLA & 2015 & & 0,965843633 & 0,982006771 & - \\
\hline BANCOLOMBLA & 2016 & & 0,977589373 & 0,973808207 & - \\
\hline BANCOLOMBIA & 2017 & $36.695,15$ & & 0,972117914 & $27.373,98$ \\
\hline BANCOLOMBLA & 2018 & $37.601,53$ & & 0,969822293 & $27.361,27$ \\
\hline BANCOLOMBIA & 2019 & $38.518,73$ & & 0,967524487 & $27.336,94$ \\
\hline BANCOLOMBLA & 2020 & $39.664,03$ & & 0,965225516 & $27.446,69$ \\
\hline BANCOLOMBIA & 2021 & $42.387,13$ & & 0,962925923 & $28.555,09$ \\
\hline
\end{tabular}

Fuente: Elaboración propia.

Figura 4. Precio Proyectado de la Acción, para las empresas del sector bancario de Colombia (Davivienda, Banco de Bogotá y Bancolombia).

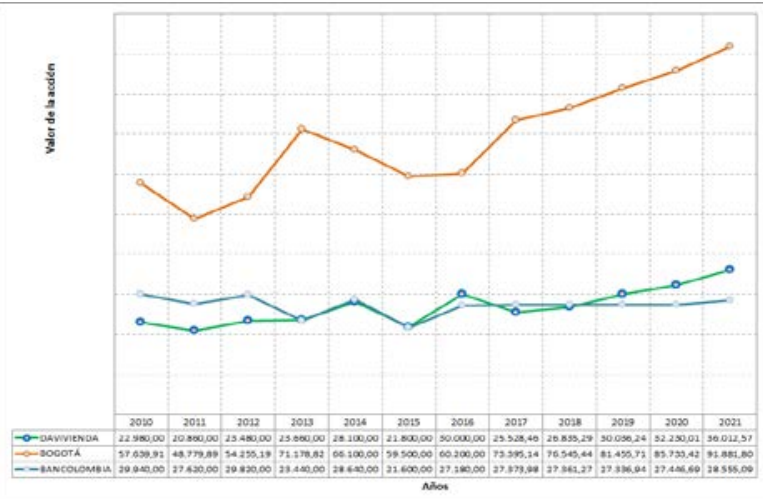

Fuente: Elaboración propia.

Para el Observatorio Financiero del CEIPA, esta técnica debe complementarse con intervalos de confianza, los cuales pueden ser generados en base a las simulaciones de Montecarlo, el cual representó un comportamiento Normal para el logaritmo natural de los promedios de los múltiplos financieros comparables, pudiendo de esta manera establecer un intervalo de confianza del 95\% para el logaritmo natural de las simulaciones realizadas. 


$$
\begin{gathered}
\text { Precio Acción Proyectado }=[\text { Li, Ls }] \\
L i=e^{\left\{\ln (\text { Precio Acción Poryectado })-Z_{\frac{\alpha}{2}} \cdot \sigma_{\text {Simulaciones. IRLC }\}}\right.} \\
L S=e^{\left\{\ln (\text { Precio Acción Poryectado })+Z_{\frac{\alpha}{2}} \cdot \sigma_{\text {Simulaciones. } I R L C}\right\}}
\end{gathered}
$$

Figura 5. Precio Proyectado de la Acción, con intervalos de confianza para las empresas del sector bancario de Colombia (Davivienda, Banco de Bogotá y Bancolombia).

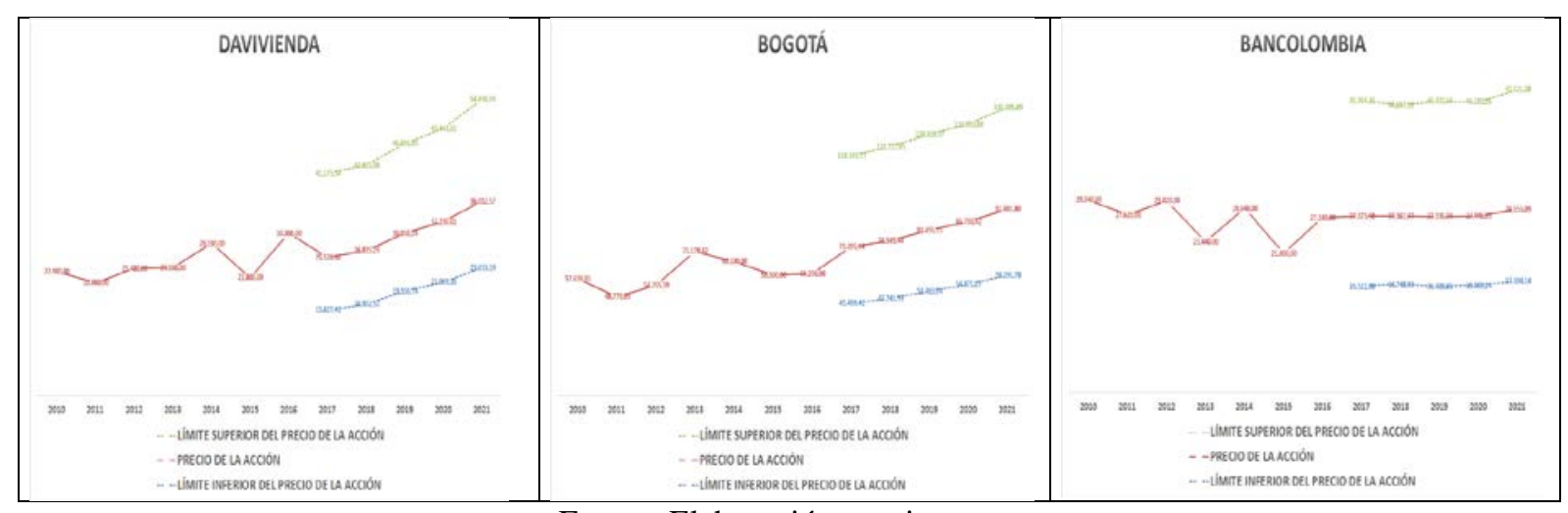

Fuente: Elaboración propia.

Dichas estimaciones exponen solamente una perspectiva estadística - financiera del precio de la acción a mediano plazo; sin embargo, estos resultados deben ser analizados por expertos cualitativos en el sector Marco-económico y bancario de Colombia. Para el Observatorio Financiero del CEIPA, es fundamental lograr una sinergia entre la valoración numérica y las perspectivas cualitativas de los expertos del entorno en el medio plazo (dentro de los intervalos de confianza anteriormente establecidos).

\section{Conclusiones.}

El análisis de cluster de los seis bancos que cotizan en la Bolsa de Valores de Colombia BVC, expone una forma interesante de análisis y segmentación fundamentado en los múltiplos financieros para mercados con economías emergentes, en donde las agrupaciones nos reflejan la evolución y crecimiento de las instituciones financieras en el tiempo, evidenciando claramente su desarrollo y potencialidades futuras, que sin duda repercutirán en el precio de la acción en el mediano plazo.

A través de este artículo, se focalizaron esfuerzos para lograr estimar el precio de la acción para tres “principales” empresas del sector bancario colombiano (Davivienda, Banco de Bogotá y Bancolombia) en el medio plazo (cinco años en el futuro), mediante la técnica de múltiplos financieros comparables, simuladas a través de distribuciones probabilísticas, con el uso de la aplicación Risk Simulator y proyectadas con el uso de un Modelo lineal dinámico Bayesiano.

Analizando estadísticamente información financiera extraída de los estados financieros auditados y publicados por Bloomberg para las empresas del sector bancario de Colombia (Davivienda, Banco de Bogotá y Bancolombia), enfocado en variables puntuales como el patrimonio, beneficio neto, ingreso, número de acciones, precio de la acción en el mercado secundario de la Bolsa de Valores de Colombia (BVC), EBITDA, EBITDA antes de provisión y pasivo, fue posible establecer una estructura de valoración mediante los múltiplos financieros derivados. 
Con este Valor Teórico, según múltiplos financieros, se procedió a realizar un análisis de sensibilidad y generar un Nuevo Valor Teórico (NVT), originado con la simulación de Montecarlo del logaritmo natural a través del Risk Simulator, y mediante la distribuciones probabilísticas de Log Normal para patrimonio, ingresos, EBITDA y pasivo (como comportamiento del retorno de una inversión según el interés compuesto para el ámbito financiero), la distribución uniforme para el beneficio y el precio de la acción de las empresas (a fin de contemplar los máximos niveles de variabilidad, sin ningún tipo de estimación ni patrón predeterminado), y distribución triangular para el EBITDA antes de provisión (distribución para ambientes de incertidumbre con escenarios optimista, pesimista y más probable) (Herrerías, Callejón y Herrerías, 2014) todos comparables en el mercado secundario de la Bolsa de Valores de Colombia.

Una vez establecido el Nuevo Valor Teórico, se determinó el proceso de predicción futura de la información para las variables iniciales de los estados financieros, fundamentándose en la información originalmente extraída de Bloomberg, determinando los Modelos Estadísticos de Regresión Lineal para predecir la tendencia de las variables de patrimonio, ingreso, EBITDA y pasivo; mientras que para el valor del beneficio, el precio de la acción y el EBITDA antes de provisión, como se poseen altos niveles de variabilidad y amplia fluctuación, se utilizaron los procesos estocásticos exponenciales (caminata aleatoria). Una vez realizado este aspecto, se realizaron cinco mil simulaciones de Montecarlo, bajo las distribuciones probabilísticas asociadas a los términos financieros anteriormente descritos, y valorando como variable de salida el logaritmo natural de los promedios de los múltiplos financieros descritos.

Con esta información, fue posible predecir el comportamiento del Nuevo Valor Teórico tanto a nivel histórico como futuro, generando un múltiplo comparativo con el Precio Real (Pr) de la acción en el mercado secundario de la BVC, creando el Indicador Relativo Logarítmico Comparativo (IRLC). Luego, este indicador, calculado para cada empresa, fue modelado mediante un Modelo Lineal Dinámico Bayesiano, generando un comportamiento de ajuste automático en el tiempo, que permitirá ajustar los valores futuros del NVT para predecir el valor de la acción en el tiempo, así como también un intervalo de confianza de 95\% que dependería de la variabilidad registrada en las simulaciones de Montecarlo.

Este artículo expone al lector, una opción de valoración para el precio de la acción de una entidad financiera en el mercado secundario de una economía emergente, aspecto que es posible mediante el uso de múltiplos financieros, tanto de la empresa para analizar como de las empresas comparables que siempre serán, como se sabe, competidoras en el mismo mercado. Bajo este escenario, si bien es cierto que los múltiplos financieros utilizados se fundamentaron en patrimonio, beneficio, ingreso, acciones en circulación, precio de la acción en el mercado secundario, EBITDA, EBITDA antes de provisión y pasivo, los resultados desde el año 2010 hasta el año 2016, comprobaron que también es necesario tomar en consideración el comportamiento de todas las variables mediante un análisis de cluster o conglomerados, y que si bien es cierto, el sector bancario de Colombia puede dividirse en "Bancos Grandes" (como Davivienda, Banco de Bogotá y Bancolombia), y “Bancos Pequeños” (como Banco de Occidente, AV villas y BBVA), es posible siempre unificar y comparar estadísticamente escenarios similares de comportamiento mediante agrupaciones de cluster.

Para muchos financieros, la cantidad de supuestos puede ser amplia. Lo interesante de este artículo radica en que cada fase ha sido realizada y estructurada con base a la información histórica de los estados financieros y balances generales de las empresas del sector bancario de Colombia, extraído directamente desde Bloomberg, sin descuidar los análisis fundamentales en función a los pasivos; todo ello, con la finalidad de documentar un procedimiento de predicción que pueda ser extensible a otros sectores, siempre y cuando puedan ser financieramente comparables mediante múltiplos y técnicas estadísticas y de simulación novedosas. 
Finalmente, podemos concluir que no es posible desligar la valoración numérica de las perspectivas cualitativas de los expertos del entorno en el mediano plazo. Es por ello, que este artículo científico sólo refleja una parte de la valoración, aspecto que posteriormente es complementado por el Observatorio Financiero del CEIPA, para brindar estimaciones más en consonancia y precisas con la realizad del entorno macroeconómico colombiano y en especial del sector bancario.

\section{Referencias}

Ariza, J. (2014). Valoración de bancos, un análisis crítico. Universidad Pontificia Comillas. Recuperado de: https://repositorio.comillas.edu/jspui/bitstream/11531/237/1/TFG000311.pdf

Beaver, W., \& Morse, D. (1978). What determines price-earnings ratios? Financial Analysts Journal, 34(4), 65-76.

Berk, J., \& De Marzo, P. (2008). Finanzas Corporativas. México. Pearson Educación.

Boada, A. (2016). La importancia de los Supuestos y sus limitaciones en los Métodos de Valoración Empresarial: Flujos de Caja Descontado vs Múltiplos Financieros Comparables. Memorias del II Congreso Arbitrado Internacional de Finanzas. Bucaramanga. Colombia.

Boada, A., \& Gallego, N. (2016). Uso del Risk Simulator ${ }^{\circledR}$ como herramienta para valoración de múltiplos comparables. Caso empresas sector eléctrico Colombia. En Memorias del VI Congreso Arbitrado Internacional Investigación Financiera FIMEF. México.

Boson, E., Cortijo, V., \& Flores, F. (2009). Análisis de Estados Financieros. Fundamentos Teóricos y Casos Prácticos. España. Pearson Educación.

Damodaran, A. (2006). Damodaran on Valuation: security analysis for investmen and corporate finance. Hoboken, NJ: Wiley.

Demirakos, E., Strong,N., \& Walker, M. (2004). What valuation models do analysts use? Accounting Horizons, 18(4), 221-240.

Fernández, P. (2008). Métodos de valoración de empresas. IESE Business School Universidad de Navarra. Recuperado de: http://www.aempresarial.com/asesor/adjuntos/metodos_de_valorizacion_de_empresas.p $\mathrm{df}$

García, O. (2003). Valoración de Empresas, Gerencia del Valor y EVA. Editorial: Cali-Prensa Moderna.

García, Y., \& García, J. (2006) Revisión bibliográfica de la evidencia empírica de los modelos multifactoriales de valoración de activos financieros, Cuadernos de Economía, XXV(44), 197-224.

Guijaro, F., \& Moya, I. (2007). Valoración de empresas cotizadas en bolsa: una revisión metodológica de los modelos factoriales. Decisiones Financieras Empresariales. pp. 148161 Recuperado de: https://dialnet.unirioja.es/descarga/articulo/2486222.pdf. 
Gutiérrez, H., \& Zhang, H. (2009). Análisis bayesiano para la diferencia de dos proporciones usando R. Revista de métodos cuantitativos para la economía y la empresa, 8, 50-70.

Herrerías, J., Callejón, J., \& Herrerías, R. (2014). Comparación de la distribución triangular, con una distribución tipo I de Pearson, como modelos para los problemas en ambiente de riesgo e incertidumbre. XXII Jornadas ASEPUMA - X Encuentro Internacional. Anales de ASEPUMA n ${ }^{\circ}$ 22, 1301_1-1301_14.

Herrerías, R., \& Herrerías, J. (2009). El modelo probabilístico rectangular-triangular. Aplicación a la tasación de fincas rústicas. XVII Jornadas ASEPUMA - V Encuentro Internacional. Anales de ASEPUMA n ${ }^{\circ}$ 19,0801_1-0801_18.

Land, J., \& M. Lang. (2002). Empirical evidence on the evolution of international earnings. The Accounting Review, 77(Supplement), 115-133.

Lind, D. (2012). Estadística aplicada a los negocios y la economía. México: McGraw Hill.

Londoño, C.A., Correa, J. C., \& Lopera, M. (2014). Estimación bayesiana del valor en riesgo: una aplicación para el mercado de valores colombiano. Cuadernos de Economía, 33(63), 635-678.

Parra, A. (2013). Valoración de empresas: Métodos de valoración. Contexto, 2, 87-100.

Pericchi, L. (2002). Análisis de Decisión, Inferencia y Predicción Estadística Bayesiana. Universidad Simón Bolívar. Edición corregida.

Sandoval, J. (2005). Distribución Lognormal. Recuperado de: https://archive.geogebra.org/en/.../ejemplo_de_la_distribucion_log_normal.html

West, M., \& Harrison, J. (1989). Bayesian Forecasting and Dynamic Models. Springer - Verlag.

Zarowin, P. (1990). What determines price-earnings ratios: Revisited. Journal of Accounting, Auditing and Finance, 5(3), 439-457. 